PROCEEDINGS OF THE

AMERICAN MATHEMATICAL SOCIETY

Volume 128, Number 10, Pages 3091-3097

S 0002-9939(00)05360-0

Article electronically published on May 2, 2000

\title{
INCOMPRESSIBLE SURFACES IN HANDLEBODIES AND CLOSED 3-MANIFOLDS OF HEEGAARD GENUS 2
}

\author{
RUIFENG QIU
}

(Communicated by Ronald A. Fintushel)

\begin{abstract}
In this paper, we shall prove that for any integer $n>0,1$ ) a handlebody of genus 2 contains a separating incompressible surface of genus $n, 2)$ there exists a closed 3-manifold of Heegaard genus 2 which contains a separating incompressible surface of genus $n$.
\end{abstract}

\section{IntRoduction}

Let $M$ be a 3-manifold, and let $F$ be a properly embedded surface in $M . F$ is said to be compressible if either $F$ is a 2 -sphere and $F$ bounds a 3 -cell in $M$, or there exists a disk $D \subset M$ such that $D \cap F=\partial D$, and $\partial D$ is nontrivial on $F$. Otherwise $F$ is said to be incompressible.

W. Jaco (see [3]) has proved that a handlebody of genus 2 contains a nonseparating incompressible surface of arbitrarily high genus, and asked the following question.

Question A. Does a handlebody of genus 2 contain a separating incompressible surface of arbitrarily high genus?

In the second section, we shall give an affirmative answer to this question. The main result is the following.

Theorem 2.7. A handlebody of genus 2 contains a separating incompressible surface $S$ of arbitrarily high genus such that $|\partial S|=1$.

If $M$ is a closed 3-manifold of Heegaard genus 1, then $M$ is homeomorphic to either a lens space or $S^{2} \times S^{1}$. In the third section, we shall prove that the Heegaard genus of a closed 3-manifold $M$ does not limit the genus of an incompressible surface in $M$. The main result is the following.

Theorem 3.10. For any integer $n>0$, there exists a closed 3-manifold of Heegaard genus 2 which contains a separating incompressible surface of genus $n$.

If $X$ is a manifold, we shall denote by $\partial X$ the boundary of $X$, and by $|\partial X|$ the number of components of $\partial X$. If $F\left(x_{1}, \ldots, x_{n}\right)$ is a free group, and $y$ is an element

Received by the editors September 20, 1996 and, in revised form, November 6, 1998.

2000 Mathematics Subject Classification. Primary 57N10.

Key words and phrases. Heegaard genus, incompressible surface.

This research was supported in part by the National Natural Science Foundation of China.

(C)2000 American Mathematical Society 


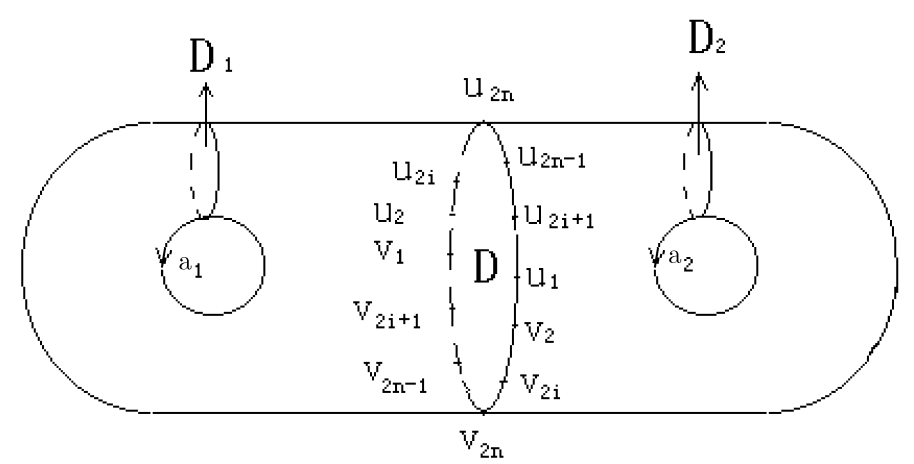

FiguRE 1.

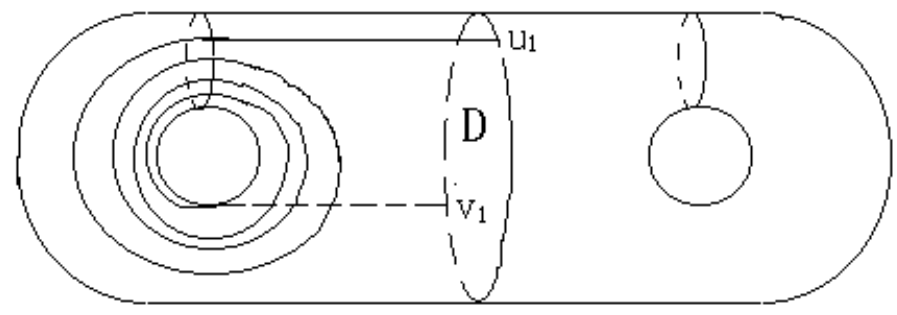

FiguRE 2.

in $F$, we shall denote by $L(y)$ the minimal length of $y$ with respect to the basis $x_{1}, \ldots, x_{n}$.

Some examples answering Jaco's question have also been given by Hugh Howards in "Generating disjoint incompressible surfaces", preprint, 1998.

\section{INCOMPRESSIBLE SURFACES IN HANDLEBODIES}

Let $H_{2}$ be a handlebody of genus 2 , and let $\left(D_{1}, D_{2}\right)$ be a set of basis disks of $H_{2}$. Let $D$ be a separating disk of $H_{2}$ such that $D_{1}$ and $D_{2}$ lie on opposite sides of $D$. Suppose that $u_{1}, \ldots, u_{2 n}, v_{1}, \ldots, v_{2 n}$ are $4 n$ points on $\partial D$ as in Figure 1 .

Suppose that $u_{1} v_{1}, \ldots, u_{2 n} v_{2 n}$ are $2 n$ arcs on $\partial H_{2}$ such that

1) $u_{1} v_{1}$ is as in Figure 2

2) $u_{2 i} v_{2 i-1}$ and $u_{2 i-1} v_{2 i}$ are as in Figure 3 ,

3) $u_{2 i+1} v_{2 i}$ and $u_{2 i} v_{2 i+1}$ are as in Figure 3, and

4) $u_{k} v_{k}$ is the union of $u_{k} v_{k-1}, v_{k-1} u_{k-1}$ and $u_{k-1} v_{k}$.

Then $u_{i} v_{i} \subset u_{i+1} v_{i+1}$.

Suppose that $N_{k}=u_{k} v_{k} \times B_{k}$, where $B_{k}$ is a half disk in $H_{2}$ (as in Figure 4) such that

1) $\left\{u_{i}\right\} \times B_{k} \cup\left\{v_{i}\right\} \times B_{k} \subset D(i \leq k)$, and

2) if $x \in u_{k} v_{k}$, then $\{x\} \times B_{i} \subset \operatorname{int}\left(\{x\} \times B_{k}\right)(i>k)$.

Let $C_{0}=D-\bigcup_{i=1}^{k}\left(\left\{u_{i}\right\} \times B_{i}\right)-\bigcup_{i=1}^{k}\left(\left\{v_{i}\right\} \times B_{i}\right)$, and $D_{0}=\bar{C}_{0}$. Let $C_{i}=$ $\partial\left(u_{i} v_{i} \times B_{i}\right)-\partial H_{2}-\left\{u_{i}\right\} \times B_{i}-\left\{v_{i}\right\} \times B_{i}$, and $D_{i}=\bar{C}_{i}$, where $1 \leq i \leq k$. Let $S_{k}=\bigcup_{i=0}^{k} D_{i}$. Then $S_{k}$ is a properly embedded surface in $H_{2}(1 \leq k \leq 2 n)$. Let 


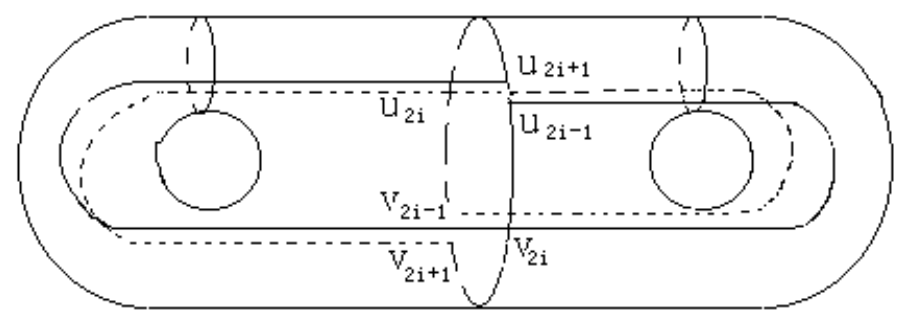

FiguRe 3.

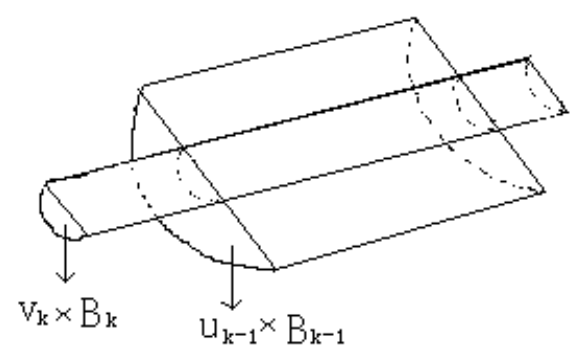

FigURE 4.

$A_{i}=D_{0} \cup D_{i}(i>0)$. Then $A_{i}$ is an annulus. Hence $S_{k}$ is a union of $k$ annuli $A_{1}, \ldots, A_{k}$ along $D_{0}$.

Lemma 2.1. $\left|\partial S_{2 k-1}\right|=2$ and $\left|\partial S_{2 k}\right|=1(1 \leq k \leq n)$.

Proof. It is clear that $\left|\partial S_{1}\right|=2$. Since $u_{2}$ and $v_{2}$ lie in the two distinct components of $\partial S_{1}$, by construction, $\left|\partial S_{2}\right|=1$. We can prove that $\left|\partial S_{2 k-1}\right|=2$ and $\left|\partial S_{2 k}\right|=1$ by induction, for $1 \leq k \leq n$.

Lemma 2.2. The genus of $S_{2 n}$ is $n$.

Proof. By construction, $S_{2 n}$ is a union of $2 n$ annuli $A_{1}, \ldots, A_{2 n}$ along $D_{0}$. Hence $\pi_{1}\left(S_{2 n}\right)=F\left(x_{1}, \ldots, x_{2 n}\right)$, where $x_{i}$ is represented by the core of $A_{i}$. Since $\left|\partial S_{2 n}\right|=$ 1 , the genus of $S_{2 n}$ is $n$.

Let $i: S_{2 n} \longrightarrow H_{2}$ be the inclusion map, and $i_{*}: \pi_{1}\left(S_{2 n}\right) \longrightarrow \pi_{1}\left(H_{2}\right)$ be the map induced by $i$. Suppose that $a_{1}$ and $a_{2}$ are the two generators of $\pi_{1}\left(H_{2}\right)$ shown in Figure 1 Then by construction we have

$$
\begin{aligned}
& i_{*}\left(x_{1}\right)=a_{1}^{4}, \\
& i_{*}\left(x_{2}\right)=a_{2}^{-1} a_{1}^{-4} a_{2}^{-1}, \\
& \cdot \\
& \cdot \\
& i_{*}\left(x_{2 i}\right)=a_{2}^{-1}\left(a_{2} a_{1}\right)^{1-i} a_{1}^{-4}\left(a_{1} a_{2}\right)^{1-i} a_{2}^{-1}, \\
& i_{*}\left(x_{2 i+1}\right)=\left(a_{1} a_{2}\right)^{i} a_{1}^{4}\left(a_{2} a_{1}\right)^{i}, \\
& \cdot \\
& \cdot \\
& i_{*}\left(x_{2 n}\right)=a_{2}^{-1}\left(a_{2} a_{1}\right)^{1-n} a_{1}^{-4}\left(a_{1} a_{2}\right)^{1-n} a_{2}^{-1} .
\end{aligned}
$$


Let $y$ be a nontrivial element of $\pi_{1}\left(S_{2 n}\right)$. Then $y=\prod_{i=1}^{m} b_{i}$, where $b_{i}=$ $\prod_{j=1}^{m_{i}} b_{i j}^{p_{i j}}, b_{i j} \in\left\{x_{1}, x_{2}, \ldots, x_{2 n}\right\}$ and $m_{i} \geq 1$, such that

1) $p_{i j} \neq 0$,

2) for each $i, 1 \leq i \leq m$, either all the $b_{i j}$ 's $\left(1 \leq j \leq m_{i}\right)$ belong to $\left\{x_{1}, x_{3}, \ldots\right.$, $\left.x_{2 n-1}\right\}$ or they all belong to $\left\{x_{2}, x_{4}, \ldots, x_{2 n}\right\}$,

$3)$ the $b_{i j}$ 's belong to $\left\{x_{1}, x_{3}, \ldots, x_{2 n-1}\right\}$ if and only if the $b_{i+1 j}$ 's belong to $\left\{x_{2}, x_{4}, \ldots, x_{2 n}\right\}$, and

4) $b_{i j} \neq b_{i j+1}^{ \pm 1}$.

Lemma 2.3. If $b_{i j} \in\left\{x_{1}, \ldots, x_{2 n-1}\right\}$ for $j \in\left\{1, \ldots, m_{i}\right\}$, then $L\left(i_{*}\left(b_{i}\right)\right)>1$. Also, the first letter of $i_{*}\left(b_{i}\right)$ is $a_{1}$ or $a_{1}^{-1}$, and the last letter of $i_{*}\left(b_{i}\right)$ is $a_{1}$ or $a_{1}^{-1}$.

Proof. Suppose that $b_{i j}=x_{2 l_{j}+1}$, where $l_{j} \in\{0,1, \ldots, n-1\}$. Then

$i_{*}\left(b_{i j}^{p_{i j}}\right)=\left(a_{1} a_{2}\right)^{l_{j}} a_{1}^{4}\left(a_{2} a_{1}\right)^{l_{j}} \ldots\left(a_{1} a_{2}\right)^{l_{j}} a_{1}^{4}\left(a_{2} a_{1}\right)^{l_{j}}$, or

$i_{*}\left(b_{i j}^{p_{i j}}\right)=\left(a_{2} a_{1}\right)^{-l_{j}} a_{1}^{-4}\left(a_{1} a_{2}\right)^{-l_{j}} \ldots\left(a_{2} a_{1}\right)^{-l_{j}} a_{1}^{-4}\left(a_{1} a_{2}\right)^{-l_{j}}$.

If $p_{i j}>0$, and $p_{i j+1}>0$, then

$i_{*}\left(b_{i j} b_{i j+1}\right)=\left(a_{1} a_{2}\right)^{l_{j}} a_{1}^{4}\left(a_{2} a_{1}\right)^{l_{j}}\left(a_{1} a_{2}\right)^{l_{j+1}} a_{1}^{4}\left(a_{2} a_{1}\right)^{l_{j+1}}$.

If $p_{i j}>0$, and $p_{i j+1}<0$, then

$i_{*}\left(b_{i j} b_{i j+1}\right)=\left(a_{1} a_{2}\right)^{l_{j}} a_{1}^{4}\left(a_{2} a_{1}\right)^{l_{j}-l_{j+1}} a_{1}^{-4}\left(a_{1} a_{2}\right)^{-l_{j+1}}$.

Since $b_{i j} \neq b_{i j+1}, l_{j} \neq l_{j+1}$. It is easy to see that the first and last letters of $i_{*}\left(b_{i}\right)$ are $a_{1}$ or $a_{1}^{-1}$, and $L\left(i_{*}\left(b_{i}\right)\right) \geq 2$.

Lemma 2.4. If $b_{i j} \in\left\{x_{2}, \ldots, x_{2 n}\right\}$, then $L\left(i_{*}\left(b_{i}\right)\right)>1$. Also, the first letter of $i_{*}\left(b_{i}\right)$ is $a_{2}$ or $a_{2}^{-1}$, and the last letter of $i_{*}\left(b_{i}\right)$ is $a_{2}$ or $a_{2}^{-1}$.

Proof. The proof of Lemma 2.4 is similar to the proof of Lemma 2.3.

Lemma 2.5. $S_{2 n}$ is incompressible in $\mathrm{H}_{2}$.

Proof. Suppose that $y$ is a nontrivial element of $\pi_{1}\left(S_{2 n}\right)$. Then $y=\prod_{i=1}^{m} b_{i}$, where $b_{i}$ satisfies the above conditions. By Lemma 2.3 and Lemma 2.4, $L\left(i_{*}(y)\right)=$ $\sum_{i=1}^{m} L\left(i_{*}\left(b_{i}\right)\right)$. Hence $L\left(i_{*}(y)\right)>1$, and $S_{2 n}$ is incompressible in $H_{2}$.

Lemma 2.6. $S_{2 n}$ is separating in $H_{2}$.

Proof. By construction, $\left[\partial S_{2 n}\right]=0$ in $H_{1}\left(\partial H_{2}\right)$. Hence $\partial S_{2 n}$ is separating on $\partial H_{2}$, and $S_{2 n}$ is separating in $\mathrm{H}_{2}$.

Theorem 2.7. A handlebody of genus 2 contains a separating incompressible surface $S$ of arbitrarily high genus such that $|\partial S|=1$.

Proof. Let $n$ be a positive integer, then $S_{2 n}$ is a separating incompressible surface in $H_{2}$ by the above argument.

Remark 1. In fact a handlebody of genus 2 also contains a separating incompressible surface $S$ of arbitrarily high genus such that $|\partial S|=2$. For example, $S_{2 n-1}$ is a separating incompressible surface of genus $n-1$ in $H_{2}$.

Remark 2. For any positive integer $n$, there exist infinitely many separating incompressible surface of genus $n$ in a handlebody of genus 2. For example, let $u_{1} v_{1}$ intersect $D_{1}$ in $m$ points where $m \geq 3$. Then by the proof of Theorem [2.7] we can obtain another separating incompressible surface of genus $n$ in $H_{2}$ by the same method as in the above construction. 
Corollary 2.8. For any integer $n>1$, a handlebody of genus $n$ contains a separating incompressible surface $S$ of arbitrarily high genus.

\section{INCOMPRESSIBLE SURFACES IN CLOSED 3-MANIFOLDS \\ of HeEgaARd genus 2}

Let $M$ be a compact 3-manifold with boundary. If $c_{1}, \ldots, c_{n}$ are disjoint simple closed curves on $\partial M$, we denote by $\tau\left(M, \bigcup_{i=1}^{n} c_{i}\right)$ the manifold obtained by attaching 2-handles to $M$ along disjoint regular neighborhoods of $c_{1}, \ldots, c_{n}$, and $M\left[c_{1}\right] \ldots\left[c_{n}\right]$ the manifold obtained by capping off possible 2 -sphere components of $\partial \tau\left(M, \bigcup_{i=1}^{n} c_{i}\right)$. If $c$ is a nontrivial simple closed curve on a toral component of $\partial M$, we denote by $M(c)$ the manifold $M[c]$. Now if $F\left(\neq S^{2}\right)$ is a separating incompressible closed surface in $\tau\left(M, \bigcup_{i=1}^{n} c_{i}\right)$, then $F$ is also a separating incompressible surface in $M\left[c_{1}\right] \ldots\left[c_{n}\right]$.

If $S$ is a properly embedded surface in $M$, we denote by $\hat{S}$ the surface obtained by capping off the boundary components of $S$ with disks in $\tau(M, \partial S)$.

Lemma 3.1. $H_{2}$ contains no closed incompressible surface.

Proof. Let $D$ be a properly embedded disk in $H_{2}$ such that $\partial D$ is nontrivial on $\partial H_{2}$. If $F$ is a closed incompressible surface, then $F$ may be isotoped to be disjoint from $D$. Hence a 3 -cell contains a closed incompressible surface, a contradiction.

Lemma 3.2. Let $M$ be a 3-manifold, and let $J$ be a simple closed curve on $\partial M$ such that $\partial M-J$ is incompressible. If $M$ has compressible boundary, then $\tau(M, J)$ is a $\partial$-irreducible manifold.

Proof. See 4, Theorem 2].

Let $H_{2}$ be a handlebody of genus 2 , and let $S$ be a separating incompressible surface of genus $n>0$ in $H_{2}$ such that $|\partial S|=1$.

Lemma 3.3. $\tau\left(H_{2}, \partial S\right)$ is a $\partial$-irreducible 3-manifold, and $\hat{S}$ is a separating closed incompressible surface of genus $n$ in $\tau\left(H_{2}, \partial S\right)$.

Proof. Suppose that $S$ separates $H_{2}$ into $H, H^{\prime}$ and $\partial S$ separates $\partial H_{2}$ into $T, T^{\prime}$, such that $\partial H=S \cup T$ and $\partial H^{\prime}=S \cup T^{\prime}$.

Claim 1. $\partial H\left(\partial H^{\prime}\right)$ is compressible in $H\left(H^{\prime}\right)$.

Proof. If $\partial H$ is incompressible in $H$, then $\partial H$ is incompressible in $H_{2}$, contradicting Lemma 3.1

Claim 2. $T\left(T^{\prime}\right)$ is incompressible in $H\left(H^{\prime}\right)$.

Proof. If $T$ is compressible in $H$, then there exists a nontrivial simple closed curve $c$ on $T$ such that $c$ bounds a disk $D$ in $H$. Since the genus of $\partial H_{2}$ is $2, T$ is a once punctured torus whose boundary is isotopic to $\partial S$. Hence $\partial S$ bounds a disk in $H_{2}$, a contradiction.

Since $\partial H\left(\partial H^{\prime}\right)$ is compressible in $H\left(H^{\prime}\right)$, and $S$ and $T\left(T^{\prime}\right)$ are incompressible in $H\left(H^{\prime}\right)$, it follows that $\tau(H, \partial S)$ and $\tau\left(H^{\prime}, \partial S\right)$ are $\partial$-irreducible 3 -manifolds by Lemma 3.2

Since $\tau\left(H_{2}, \partial S\right)=\tau(H, \partial S) \cup \tau\left(H^{\prime}, \partial S\right), \hat{S}$ is a separating incompressible closed surface in $\tau\left(H_{2}, \partial S\right)$. 
Since the genus of $\partial H_{2}$ is $2, \partial \tau\left(H_{2}, \partial S\right)$ consists of two tori, $T_{1}$ and $T_{2}$, say.

Let $M$ be a 3-manifold with one of component $T$ of $\partial M$ a torus. If $r_{1}$ and $r_{2}$ are two slopes on $T$, we shall denote by $\Delta\left(r_{1}, r_{2}\right)$ the minimal geometric intersection number among all the curves representing the slopes.

Lemma 3.4. Let $M$ be a $\partial$-irreducible 3-manifold with one component $T$ of $\partial M a$ torus, and let $F$ be a closed incompressible surface in $M$ which is not parallel to $T$. If $r_{1}$ and $r_{2}$ are two slopes on $T$ such that $F$ is compressible in $M\left(r_{1}\right)$ and $M\left(r_{2}\right)$, then either

1) $\Delta\left(r_{1}, r_{2}\right) \leq 1$, or

2) there exists a slope $r$ on $T$ such that $\Delta\left(r, r_{1}\right) \leq 1$ and $\Delta\left(r, r_{2}\right) \leq 1$.

Proof. See [6. Theorem 1].

Corollary 3.5. Let $M$ be a $\partial$-irreducible 3-manifold with one component $T$ of $\partial M$ a torus. If $F$ is a closed incompressible surface in $M$ which is not parallel to $T$, then there exists a nontrivial simple closed curve $c$ on $T$ such that $F$ is incompressible in $M(c)$.

Lemma 3.6. There exist two nonseparating simple closed curves $c_{1}$ and $c_{2}$ on $\partial H_{2}$ such that $c_{i}$ is disjoint from $\partial S$, and $\hat{S}$ is a separating incompressible surface in $H_{2}[\partial S]\left[c_{1}\right]\left[c_{2}\right]$.

Proof. Suppose that $\hat{S}$ separates $\tau\left(H_{2}, \partial S\right)$ into $M_{1}$ and $M_{2}$ such that $T_{1} \subset M_{1}$ and $T_{2} \subset M_{2}$. By the proof of Lemma 3.1, $M_{i}$ is $\partial$-irreducible. Since $\hat{S}$ is not parallel to $T_{i}$ in $M_{i}$, by Corollary 3.5 , there exists a simple closed curve $c_{i}(1 \leq i \leq 2)$ on $T_{i}$ such that $\hat{S}$ is incompressible in $M_{i}\left(c_{i}\right)$.

By an isotopy, we can suppose that $c_{i}$ is disjoint from $\partial S$. Hence $\hat{S}$ is incompressible in $H_{2}[\partial S]\left[c_{1}\right]\left[c_{2}\right]$. It is easy to see that $\hat{S}$ is separating in $H_{2}[\partial S]\left[c_{1}\right]\left[c_{2}\right]$.

Definition 3.7. Two simple closed curves $\alpha$ and $\beta$ on $\partial M$ are said to be coplanar if some component of $\partial M-\alpha \cup \beta$ is an annulus or a once punctured annulus.

Lemma 3.8. Suppose that $\alpha$ is a nonseparating curve on $\partial M$. If a separating curve $\beta$ on $\partial M$ is coplanar to $\alpha$, then $M[\alpha]=M[\beta][\alpha]$.

Proof. See [5, Lemma 5.1].

Lemma 3.9. $H_{2}[\partial S]\left[c_{1}\right]\left[c_{2}\right]=H_{2}\left[c_{1}\right]\left[c_{2}\right]$.

Proof. Since $c_{1}$ is coplanar to $\partial S$ on $\partial H_{2}$, we have $H_{2}[\partial S]\left[c_{1}\right]\left[c_{2}\right]=H_{2}\left[c_{1}\right]\left[c_{2}\right]$.

Theorem 3.10. For any integer $n>0$, there exists a closed 3-manifold $M$ of Heegaard genus 2 which contains a closed separating incompressible surface of genus $n$.

Proof. Let $\mathrm{H}_{2}$ be a handlebody of genus 2, and let $S$ be a separating incompressible surface of genus $n$ such that $|\partial S|=1$. Then $H_{2}\left[c_{1}\right]\left[c_{2}\right]$ contains a separating incompressible surface $\hat{S}$ of genus $n$, where $c_{1}$ and $c_{2}$ are disjoint nonseparating simple closed curves on $\partial H_{2}$ as in Lemma 3.6. Obviously the Heegaard genus of $H_{2}\left[c_{1}\right]\left[c_{2}\right]$ is 2 .

Corollary 3.11. Suppose that $m \geq 2$. Then for any integer $n>0$ there exists a closed 3-manifold of Heegaard genus $m$ which contains a closed separating incompressible surface of genus $n$. 
In fact there are infinitely many simple closed curves $c$ on $\partial H_{2}$ such that $\tau\left(H_{2}, c\right)$ contains a closed separating incompressible surface of genus $n$. This is shown by the following example.

Example. Let $H_{2}$ be a handlebody of genus 2. Let $S_{2 m}$ be an incompressible surface of genus $n$ constructed by the same method as the construction of $S_{2 n}$ (as in Section 2), such that $u_{1} v_{1}$ intersects $D_{1}$ in $m$ points. Then $\hat{S}_{2 m}$ is incompressible in $\tau\left(H_{2}, \partial S_{2 m}\right)$.

I am grateful to Professor R. Fintushel and the referee for their suggestions.

\section{REFERENCES}

[1] C. Gordon and R. Litherland, Incompressible planar surfaces in 3-manifolds, Topology and its Appl. 18(1984), 121-144. MR 86e:57013

[2] J. Hempel, 3-manifolds, Ann. of Math. Studies 86. Princeton Univ. Press, Princeton, New Jersey, 1976. MR 54:3702

[3] W. Jaco, Lectures on 3-manifold Topology, Published by Amer. Math. Soc., Providence, Rhode Island, 1980. MR 81k:57009

[4] W. Jaco, Adding a 2-handle to a 3-manifold, An application to Property R, Proc. A. M. S. 92(1984), 288-292. MR 86b:57006

[5] M. Scharlemann and Y-Q Wu, Hyperbolic manifold and degenerating handle additions, J. Austral. Math. Soc. (series A) 55(1993), 72-89. MR 94e:57019

[6] Y-Q Wu, Incompressibility of surfaces in syrgered 3-manifolds, Topology 31(1992), 271-280. MR 94e:57027

Department of Mathematics, Jilin University, Changchun 130023, People's Republic OF CHINA

E-mail address: qrf@mail_jlu.edu.cn 